\title{
Pharmacotherapies for Drug-Induced Liver Injury: A Current Literature Review
}

\author{
Meng $L^{1}{ }^{1}$, Qiong Luo ${ }^{1}$, Yanyan Tao ${ }^{1}$, Xin Sun ${ }^{1,2 *}$ and Chenghai Liu ${ }^{1,2,3,4 *}$ \\ ${ }^{1}$ Institute of Liver Diseases, Shuguang Hospital Affiliated to Shanghai University of Traditional Chinese Medicine, Shanghai, China, \\ ${ }^{2}$ Shanghai Key Laboratory of Traditional Chinese Clinical Medicine, Shanghai, China, ${ }^{3}$ Key Laboratory of Liver and Kidney \\ Diseases, Ministry of Education, Shanghai, China, ${ }^{4}$ Shanghai Innovation Center of TCM Health Service, Shanghai, China
}

\section{OPEN ACCESS}

Edited by:

Mercedes Robles-Díaz, University of Malaga, Spain

Reviewed by:

Andreas Benesic, Krankenhaus GmbH Weilheim-

Schongau, Germany

Fernando O. Bessone,

National University of Rosario,

Argentina

Maria Isabel Schinoni,

Federal University of Bahia, Brazil

*Correspondence:

Xin Sun

913637769@qq.com

Chenghai Liu

chenghai.liu@outlook.com

Specialty section:

This article was submitted to Gastrointestinal and Hepatic

Pharmacology,

a section of the journal

Frontiers in Pharmacology

Received: 31 October 2021 Accepted: 16 December 2021

Published: 05 January 2022

Citation:

Li M, Luo Q, Tao Y, Sun X and Liu C (2022) Pharmacotherapies for DrugInduced Liver Injury: A Current Literature Review.

Front. Pharmacol. 12:806249. doi: 10.3389/fphar.2021.806249
Drug-induced liver injury (DILI) has become a serious public health problem. For the management of DILI, discontinuation of suspicious drug or medicine is the first step, but the treatments including drugs and supporting approaches are needed. Reference to clinical patterns and disease severity grades of DILI, the treatment drugs were considered to summarize into hepatoprotective drugs (N-acetylcysteine and Glutathione, Glycyrrhizin acid preparation, Polyene phosphatidylcholine, Bicyclol, Silymarin), anticholestatic drug (Ursodeoxycholic acid, S-adenosylmethionine, Cholestyramine), immunosuppressants (Glucocorticoids) and specific treatment agents (L-carnitine, Anticoagulants). The current article reviewed the accumulated literature with evidence-based medicine researches for DILI in clinical practice. Also the drawbacks of the clinical studies involved in the article, unmet needs and prospective development for DILI therapy were discussed.

Keywords: drug-induced liver injury, acute liver failure, pharmacotherapy, hepatoprotective drug, anticholestatic drug

\section{INTRODUCTION}

With the extensive use of drugs and the continuous development and application of new drugs, the safety of drug use is widely concerned. Drug-induced liver injury (DILI) is one of the most common and serious adverse drug reactions (ADRs) (Miguel et al., 2012). DILI can be caused by various prescription or non-prescription chemical drugs, including biological agents, traditional Chinese medicine (TCM), natural medicine (NM), health care products (HP), dietary supplements (DS), their metabolites and excipients (European Association for the Study of the Liver. 2019). To date, with no specific diagnostic markers, diagnosis of DILI has been very challenging, and the pathogenesis has not been understood completely. Misdiagnosis and inappropriate treatment are quite likely resulting in unavoidable systematic errors in the efficacy of DILI interventions. In clinical practice, Roussel Uclaf Causality Assessment Method (RUCAM) and liver biopsy could help clinicians to better diagnose and differentiate DILI. Most countries and regions in the world also have summarized the empirical therapeutic strategies for DILI and formed guidelines. Actually, DILI could be categorized three clinical patterns as hepatocellular, cholestatic or mixed injury (liver damaged target cells) based on serum ALT and ALP ratio from the first available biochemical test (Chalasani Naga et al., 2021). So, the therapeutic strategies of DILI, especially therapeutic drugs, can be also classified reference to these three clinical patterns of DILI. In the article, the therapeutic agents of DILI were reviewed and summarized based on the published literature. Our search was performed in PubMed, CNKI and Web of Science, searching for the terms "drug-induced liver injury," "acute liver failure," "treatment," 
"pharmacotherapy," "protect hepatocyte," "cholagogue" and/or "trial." Clinical studies, case reports, reviews and guidelines were included. However, the published data about therapeutic drugs of DILI were heterogeneous. More well-designed studies, including control trials, are needed and a multicentre approach may be required in the future.

\section{BASAL PRINCIPLES OF DILI MANAGEMENT}

The most definitive treatment for DILI is to suspend the offending $\operatorname{drug}(\mathrm{s})$ and to avoid re-exposure (European Association for the Study of the Liver. 2019; Chalasani Naga et al., 2021). After drug withdrawal, most liver damage can recover spontaneously, with recovery time ranging from a few days to a few weeks (Devarbhavi et al., 2010). Actually, spontaneous recovery after discontinuation of the susceptible drugs or medicines is important criterion in the causality assessment. However, multiple suspicious drugs are often used together in clinical practice, it is difficult to identify which drugs or drug interactions cause liver injury. Moreover, many drugs are necessary for treatment of primary disease, and discontinuation of drugs may lead to serious consequences, which is also a major difficulty in the clinical treatment of drug-induced liver injury. Besides, small elevations in liver parameters induced by drugs may be transient in nature and may revert to baseline even when therapy is continued (such as antituberculosis drug and statin). These elevations may represent mild liver injury with spontaneous resolution or "adaptation." So far, the mechanisms potentially underlying this "adaptation phenomena" were not known (Watkins Paul, 2005; Aithal et al., 2011). Therefore, it is necessary to fully evaluate the risk of primary disease progression caused by drug withdrawal and exacerbation of liver injury caused by continued drug use. To avoid unnecessary drug withdrawal, the International Serious Adverse Events Consortium (iSAEC) recommended in 2011 the modified biochemical criteria for the identification of DILI as reaching any of the following items: 1 ) ALT $\geq 5 \times$ upper limit of normal (ULN); 2) ALP $\geq 2$ ULN, especially in patients with elevated 5 '-nucleotidase or GGT, and without bone-diseasesrelated ALP elevation; 3) ALT $\geq 3$ ULN and TBil $\geq 2$ ULN (Aithal et al., 2011). Although not clinical diagnostic criterion for DILI, it can provide a reference for treatment decisionmaking. Besides, the DILI patient should be educated and given alert card to avoid repeating the use of the offending agent or similar ones with known cross reactivity because continued exposure is associated with the development of severe or chronic liver diseases (Aithal and Day, 1999). The pharmacotherapy of DILI is particularly important, especially for patients with severe hepatitis and acute liver failure (ALF). Pharmacotherapy strategies are mainly aimed at protecting hepatocytes, scavenging free radicals and anti-oxidation, stabilizing hepatocytes membrane, detoxifying, reducing transaminase, immune regulation and so on. As we mentioned, it requires appropriate drug administration according to the clinical pattern of DILI. This part will be summarized detailedly in the following sections. In addition, patients with ALF or subacute liver failure (SALF) should consider artificial liver supporting treatment and liver transplantation (Devarbhavi et al., 2021).

Shown as Table 1, the basic principles for treating DILI are 1) identifying the offending $\operatorname{drug}(\mathrm{s})$ and prompt withdrawal before irreversible liver damage; 2) monitoring liver biochemistries, assessing risk and benefit of dubious drug(s) for primary diseases; 3 ) appropriate medication is selected reference to the clinical pattern of DILI; 4) artificial liver supporting therapy and liver transplantation for ALF/SALF patients.

\section{PHARMACOTHERAPY STRATEGIES}

It required appropriate pharmacotherapy to reduce liver damage after stopping use the offending $\operatorname{drug}(\mathrm{s})$, when serum transaminase of patients could not normalize and bilirubin remained abnormal within a long time. Reference to the clinical patterns of DILI, pharmacotherapy was considered to classify as follows: 1) hepatoprotective drugs, 2) anticholestatic drug, 3) immunosuppressants, 4) specific treatment. And their efficacy for DILI based on clinical practice evidence was summarized in Table 2.

\section{Hepatoprotective Drugs}

Hepatoprotective drugs refer to drugs that can improve liver function, promote liver cell regeneration and/or enhance liver detoxification, and there is no unified understanding in regard to its classification. According to the different mechanism of action, it can be roughly divided into detoxification drugs (eg. NAC and GSH), anti-inflammatory drugs (eg. Glycyrrhizic acid preparation), hepatocyte membrane protector (eg. PPC), antioxidant drugs (eg. Bicyclol, Silymarin) (Chinese Society of Infectious Diseases, 2014).

\section{N-Acetylcysteine and Glutathione}

$\mathrm{NAC}$ is an N-acetylated derivative of L-cysteine (L-Cys) and a precursor of Glutathione (GSH), and has the pharmacological effects of anti-oxidation, anti-inflammation, dilatation of microvessels and protection of DNA molecules (Dekhuijzen, 2004). It is the only antidote approved by the US Food and Drug Administration (FDA) in 2004 for acetaminophen (APAP)induced DILI. In an observational study from Edinburgh, intravenous NAC was claimed to be equally as effective as cysteamine and methionine and free of adverse effects (Prescott et al., 1979). Ever since, NAC has been accepted as an antidote for acetaminophen (APAP) overdose either intravenously or orally (Chiew et al., 2018). In addition, NAC can also be applied to liver injury caused by other drugs and DILI with ALF. Moosa MS et al. conducted a randomized, doubleblind, placebo-controlled trial to assess whether intravenous NAC hastens liver recovery in hospitalized adult patients with anti-tuberculosis (Anti-TB) DILI. The results revealed that NAC did not shorten time to ALT $<100 \mathrm{U} / \mathrm{L}$ in participants with AntiTB DILI, but significantly reduced length of hospital stay (Moosa et al., 2020). In another study NAC together with prednisolone 
TABLE 1 | Basal principles of DILI management.

\section{Principles}

I. Identify \& Withdrawal

II. Monitor \& Assessment

III. Appropriate medication

(according to the clinical pattern of DILI)

IV. Liver transplantation

Identifying the offending drug(s) and prompt withdrawal before irreversible liver damage Monitoring liver biochemistries, assessing risk and benefit of dubious drug(s) for primary diseases

Hepatocellular type

Cholestatic type

Specific type

Artificial liver supporting therapy and liver transplantation for ALF/SALF patients

Note: NAC, N-acetylcysteine; MglG, magnesium isoglycyrrhizinate; PPC, Polyene phosphatidylcholine; UDCA, Ursodeoxycholic acid; SAMe, S-adenosylmethionine; GCs, Glucocorticoids; $L C$, L-carnitine; $A L F$, acute liver failure; $S A L F$, subacute liver failure.

when used in cases of severe idiosyncratic DILI due to fupirtine (central acting non-opiod analgesic) showed a significant improvement in ALT, AST and INR within 2 weeks compared to those who were not treated with NAC (Borlak et al., 2018). Furthermore, it is reported that NAC is benefit for tyrosine kinase inhibitors and norfloxacin-induced acute liver injury (Elliott et al., 2016; Patel et al., 2019). As shown in a prospective controlled study by an American ALF research group, which was conducted in 24 medical centers for 8 years in 173 patients with ALF caused by non-APAP agents, NAC could improve the survival rate of patients who were at the early stage of DILIrelated ALF and had not undergone liver transplantation (Lee et al., 2009). Although the logistic regression analysis in their study did not show significant results, the NAC use also appeared to be associated with improved transplant free survival (Reuben, A. et al., 2010). However, a randomized, controlled treatment trial in children with ALF caused by non-APAP agents did not support a therapeutic role for NAC (Squires et al., 2013). Therefore, NAC is not recommended to treat children ALF caused by non-APAP agents, particularly pediatric patients of less than 2 years (Chalasani Naga et al., 2021). In a word, NAC was recommended for use in patients with early ALF, but was not recommended in children.

\section{Glycyrrhizin Acid Preparation}

Glycyrrhizin acid, extracted from the roots of the plant Glycyrrhiza glabra, is a triterpenoid compound, also known as glycyrrhizin for its sweet taste. It is hydrolyzed in vivo by glucuronidase to glucuronic acid and glycyrrhizin acid. At present, Glycyrrhizin acid preparation have been commonly used in the treatment of DILI in clinical application around the world, mainly including compound glycyrrhizin tablets, magnesium isoglycyrrhizinate (MgIG) and other drugs (Chen and Sun, 2011). It plays an anti-oxidation, anti-inflammation and hormone-like role in DILI treatment and protects liver against inflammatory damage. In China, Mao YM and coworkers carried out a multi-center, randomized, double-blind, multi-dose, positive drug parallel control clinical study of MgIG in the treatment of chronic liver disease (CLD) with elevated ALT among 412 patients including DILI. The results showed that MgIG can significantly reduce ALT and AST levels in patients, and the total effective rate was high $(72.22 \%, 73.53 \%)$. Meanwhile, there was no obvious recurrence after withdrawal, and the incidence of adverse reactions was low $(1.85 \%, 1.18 \%)$
(Mao et al., 2009). In 2015, MgIG has been approved by Chinese Food and Drug Administration (CFDA) to treat acute DILI, including the patterns of acute hepatocellular injury and mixed liver injury with significantly elevated serum ALT. Mao YM and coworkers then carried out a randomized, double-blind, multidoses, active drug controlled, multi-center phase II trial involved 174 patients to assess the safety and efficacy of MgIG, as compared to tiopronin, a standard therapy for DILI in China and of which action was similar to NAC (Chiew et al., 2018). It was shown that both low-dose and high-dose MgIG lowered ALT level even at early stage of study drug administration. When compared with tiopronin (61.02\%), the proportions of ALT normalization at week 4 were significantly greater in low dose MgIG (84.75\%) and high dose MgIG (85.71\%), respectively (Wang et al., 2019). Tang L et al. completed a phase III clinical trial of MgIG in the treatment of acute drug-induced liver injury induced by antineoplastic drugs. A total of 55 patients with antineoplastic DILI were treated with MgIG or tiopronin for 2 weeks respectively. The results revealed that after 1 week of treatment, the overall effective rate of $\mathrm{MgIG}$ group was significantly higher than that of the positive control group. After 2 weeks of treatment, the normalization rate of ALT and AST in MgIG group was significantly higher than that of tiopronin, but bilirubin levels were not mentioned. There was no statistical significance in the incidence of adverse reactions between the two groups (Tang et al., 2012).

\section{Polyene Phosphatidylcholine}

PPC is refined from phospholipids, which is extracted from soybeans. Accounting for about 52\%, diacylphospholipidcholine (DLPC) is the main active ingredient. It can provide endogenous phospholipids to repair damaged liver cell and organelle membrane, so as to restore membrane function and increase the fluidity and stability of cell membrane, which protects from liver diseases caused by a variety of factors (Committee of the treatment with polyenphosphatidylcholine in patients with liver diseases, 2017). Lei $X$ found that the ALT recurrence rate of PPC treatment in DILI was the same as MgIG (34.97\%) through reanalysis of data from a multicenter retrospective cohort study in China (Lei et al., 2021). A single-center clinical study of 78 patients with anti-TB DILI found that PPC significantly increased serum heme oxygenase-1 (HO-1) and superoxide dismutase (SOD) levels and thus reduced oxidative stress 
TABLE 2 | Pharmacotherapy strategies of DILI.

\begin{tabular}{|c|c|c|c|c|c|}
\hline Drugs Classification & Drugs name & Author \& Year & Type of Study & Culprit drug & Outcomes/Conclusion \\
\hline Hepatoprotective & NAC and GSH & Prescott et al. (1979) & Comparative Study & Paracetamol & LFP, SVI \\
\hline \multirow[t]{20}{*}{ Drugs } & & Moosa et al. (2020) & $\begin{array}{l}\text { Randomized, double-blind, } \\
\text { placebo-controlled trial }\end{array}$ & Anti-tuberculosis drug & SHS \\
\hline & & Borlak et al. (2018) & Retrospective cohort study & Flupirtine & LFP \\
\hline & & Patel et al. (2019) & Case Reports & $\begin{array}{l}\text { Tyrosine Kinase } \\
\text { Inhibitor }\end{array}$ & LFP \\
\hline & & Elliott et al. (2016) & Case Reports & Norfloxacin & LFP \\
\hline & & Lee et al. (2009) & Randomized Controlled Trial & Non-APAP agents & LFP, SVI \\
\hline & & Reuben et al. (2010) & Prospective observational study & Multiple drugs & LFP, SVI \\
\hline & & Squires et al. (2013) & Randomized Controlled Trial & Non-APAP agents & NSD \\
\hline & $\begin{array}{l}\text { Glycyrrhizin acid } \\
\text { preparation }\end{array}$ & Wang et al. (2019) & $\begin{array}{l}\text { Randomized, double-blind, } \\
\text { controlled trial }\end{array}$ & Multiple drugs & LFP \\
\hline & & Tang et al. (2012) & Phase III clinical trial & Anti-neoplastic drugs & LFP \\
\hline & Bicyclol & Naiqiong et al. (2017) & Randomized Controlled Trial & Statin & LFP \\
\hline & & Chen et al. (2018) & Comparative Study & Anti-tuberculosis drug & LFP \\
\hline & & Li et al. (2018) & Retrospective cohort study & Anti-neuropathy drug & LFP \\
\hline & & Zhou et al. (2015) & Randomized controlled study & Anti-psoriasis drug & LFP \\
\hline & Polyene & Lei et al. (2021) & Literature analysis & Multiple drugs & LFP \\
\hline & phosphatidylcholine & Liu et al. (2021) & Retrospective controlled study & Anti-tuberculosis drug & LFP \\
\hline & Silymarin & Enjalbert et al. (2002) & Retrospective analysis & Toadstool & SVI \\
\hline & & Grabhorn et al. (2013) & Retrospective controlled study & Toadstool & SVI \\
\hline & & Masoumeh et al. (2015) & $\begin{array}{l}\text { Open-label, randomized clinical } \\
\text { trial }\end{array}$ & Anti-convulsive drugs & LFP \\
\hline & & Majid. et al. (2019) & $\begin{array}{l}\text { Randomized, double-blind } \\
\text { clinical trial }\end{array}$ & Anti-tuberculosis drug & NSD \\
\hline & & Zhao et al. (2021) & Retrospective cohort study & Multiple drugs & LFP \\
\hline \multirow[t]{11}{*}{ Anticholestatic drugs } & Ursodeoxycholic acid & Lang et al. (2019) & Prospective pilot study & $\begin{array}{l}\text { Anti-mycobacterial } \\
\text { infections drugs }\end{array}$ & LFP \\
\hline & & Sun et al. (2015) & Retrospective controlled study & Multiple drugs & LFP \\
\hline & & Asgarshirazi et al. (2015) & Prospective observational study & Anticonvulsants & LFP \\
\hline & & Saito et al. (2016) & Retrospective controlled study & Anti-tuberculosis drug & NSD \\
\hline & & Bessone et al. (2021) & Review & Multiple drugs & UK \\
\hline & & $\begin{array}{l}\text { Robles-Díaz Mercedes. } \\
\text { et al. (2021) }\end{array}$ & Systematic review & Multiple drugs & UK \\
\hline & S-adenosylmethionine & Almasio et al. (1990) & Review & Multiple drugs & LFP \\
\hline & & Perlamutrov et al. (2014) & Prospective observational study & $\begin{array}{l}\text { Immune-suppressive } \\
\text { therapy }\end{array}$ & LFP \\
\hline & & $\begin{array}{l}\text { Hayashi and Fontana. } \\
(2014)\end{array}$ & Prospective observational study & Chemotherapy & LFP \\
\hline & & Santini et al. (2003) & Prospective observational study & Chemotherapy & LFP \\
\hline & Cholestyramine & Stine and Lewis. (2016) & Review & Leflunomide & UK \\
\hline \multirow[t]{8}{*}{ Immunosuppressants } & Glucocorticoids & Hou et al. (2012) & Retrospective cohort study & Multiple drugs & LFP, SHS \\
\hline & & Hu et al. (2016) & Retrospective cohort study & Multiple drugs & LFP, SHS, SVI \\
\hline & & Sundaram et al. (2020) & Case Reports & Imatinib, cephalexin & LFP \\
\hline & & $\begin{array}{l}\text { Herrero-Herrero and } \\
\text { García-Aparicio. (2010) }\end{array}$ & Case Reports & Amoxicillin-clavulanate & LFP \\
\hline & & Karkhanis et al. (2014) & Retrospective cohort study & Multiple drugs & NSD \\
\hline & & Wan et al. (2019) & Prospective observational study & Multiple drugs & NSD \\
\hline & & Pang et al. (2018) & Retrospective controlled study & Multiple drugs & NSD \\
\hline & & Hu and Xie. (2019) & Review & Multiple drugs & UK \\
\hline \multirow{4}{*}{$\begin{array}{l}\text { Specific drugs } \\
\text { treatment }\end{array}$} & L-carnitine & Bohan et al. (2001) & Retrospective cohort study & Valproic acid & LFP \\
\hline & Anticoagulants & Wang et al. (2016) & Retrospective cohort study & Gynura segetum & SVI \\
\hline & & Richardson et al. (1998) & $\begin{array}{l}\text { Retrospective observational } \\
\text { study }\end{array}$ & HSCT & LFP, SVI \\
\hline & & Richardson et al. (2016) & $\begin{array}{l}\text { historically controlled, } \\
\text { multicenter, open-label, phase iii } \\
\text { trial }\end{array}$ & HSCT & LFP, SVI \\
\hline
\end{tabular}

Note: LFP, Liver function parameters improvement (including ALT, AST, ALP, GGT, TBIL, INR, or PT, etc.); SHS, Shorter hospital stay; SVI, Survival improvement (including the extension of survival time or improved survival rates); NSD, No significant difference; UK, Unknown or remain controversial. 
responses (Liu et al., 2021). Moreover, PPC was often used as a control or combination agent in many clinical studies of DILI, demonstrating reliable efficacy in the treatment of DILI. Although only widely used in China so far, PPC has the potential clinical application value and is expected to be studied in more high-quality clinical studies in future.

\section{Bicyclol}

Bicyclol (4, 40-dimethoxy-5, 6, 50, 60-dimethylene-dioxy-2, 20dicarboxylate biphenyl) is the first chemical new drug independently developed by China and used to treat inflammatory liver injury. It has been recommended by Russian physicians' clinical guidelines for the treatment of DILI (Expert Committee on Clinical Application of Bicyclol, 2020). Its pharmacological activity mainly lies in its inhibition of the expression and activity of NF- $\kappa B$, IL- $1 \beta$, IL-18, TNF- $\alpha$, TGF- $\beta 1$ and other inflammatory regulatory factors induced by liver injury, as well as the production of reactive oxygen species (ROS) and nitric oxide (NO), reducing consumption of antioxidants such as GSH (Liu et al., 2017). Many clinical studies have shown that bicyclol demonstrated a good protective effect on the liver during acute and chronic liver injuries caused by a variety of chemical poisons, alcohols, and others agents (Expert Committee on Clinical Application of Bicyclol, 2020). Naiqiong W et al. conducted a multicenter and randomized controlled trial of 168 patients to explore the efficacy and safety of oral bicyclol versus polyene phosphatidylcholine in the treatment of statin-induced liver injury. After 2 and 4 weeks of treatment, the reduction of ALT in bicyclol ( $25 \mathrm{mg}$ tid.po.) group was significantly higher than that of the polyene phosphatidylcholine (456 mg tid.po.) group, and there was no significant difference in the incidence of adverse reactions between the two groups (Naiqiong et al., 2017). Chen Y et al. evaluated the pharmacoeconomics of three therapeutic schemes in treating Anti-TB DILI of 225 newly treated TB patients. The results revealed that the efficacy of bicyclol in the treatment of Anti-TB DILI was superior to silybin and diammonium glycyrrhizinate, with good safety (Chen et al., 2018). In a meta-analysis involving 7 RCTs, it was also found that bicyclol had a good therapeutic effect on Anti-TB DILI, but all these 7 included studies were of low quality with high heterogeneity, and plausible publication bias was found (Wang et al., 2018). In addition, bicyclol had been reported to be effective for anti-neuropathy DILI (Li et al., 2018) and anti-psoriasis DILI (Zhou et al., 2015). More clinical studies are needed to provide sufficient evidence of its efficacy.

\section{Silymarin}

Silymarin is a flavonoid isolated for the first time in 1968 from the seed extract of milk thistle plant which is mainly a mixture of lignin-derived flavonols, including silybin, silydianin, silychristin, and isosilybin (Tittel and Wagner, 1977; Borah et al., 2013) with silybin is the most potent constituent (Committee of experts on silymarin therapy in patients with liver disease, 2016). Such drugs can maintain the fluidity of liver cell membrane through antilipid peroxidation and enhance the resistance of liver cell membrane to various injury factors, so as to protect against drug-induced liver injury (Chen and Sun, 2011). Enjalbert et al. (2002) reviewed the clinical data of 2,000 patients with liver injury caused by toadstool in 20 years and found that silymarin could significantly reduce the mortality rate of patients with toadstool poisoning (5.8\%), while that of those who did not use silymarin was as high as $14.1 \%$. There is also clinical evidence that early administration of silymarin can improve clinical outcome in children with toadstool poisoning (Grabhorn et al., 2013). Except treating liver injury caused by toxic substances, more importantly, there are also clinical studies indicating that it has the potential to treat DILI. Masoumeh Asgarshirazi et al. prospectively studied the efficacy and tolerance of Silymarin and Ursodeoxycholic acid (UDCA) in 46 anticonvulsive drugs induced hypertransaminasemia. The results showed that after 1 month of Silymarin treatment, ALT changes were significantly better than those in UDCA group, without obvious side effects (Masoumeh et al., 2015). A multicenter, retrospective, non-intervention cohort study conducted by Hong Zhao et al. revealed that Silybin alone improved liver function in patients with DILI (Zhao et al., 2021). However, at the same time, some studies have cast doubt on its therapeutic efficacy. A randomized double blind clinical trial enrolled 55 cases with anti-tuberculosis drugs induced liver injury were conducted in Iran (Majid. et al., 2019). The observational measures include severity of liver injury, the duration necessary for normalization of liver function, hospital stay and adverse effects. Consequently, there was not any statistically significant difference between silymarin and placebo groups. And the author concluded that silymarin is a safe herbal medication, but no effect to treat hepatic toxicity of anti-tuberculosis drugs.

\section{Anticholestatic Drugs}

Multiple drugs have been reported to cause bile duct injury, which mostly affects the biliary epithelium of the interlobular ducts, thereby causing an inflammatory response directed at cholangiocytes. Cholestatic and mixed cholestatic and hepatocellular injury are two of the most severe manifestations of drug induced liver disease (DILD) (Bohan and Boyer, 2002). There is increasing evidence that drugs that are excreted by the liver into bile are prime candidates for producing cholestatic liver disease in the susceptible patient (Björnsson and Olsson, 2005). Many drugs target the biliary epithelium and result in "drug-induced cholangiopathy" and the "vanishing bile duct syndrome" (VBDS), characterized by loss of bile duct $>50 \%$ due to inflammatory response and necrosis of the intralobular bile duct epithelium (Padda et al., 2011; Bessone et al., 2021). Most cases of drug induced cholestasis will resolve with withdrawal of the offending medication and not develop chronic liver disease. However, persistent cholestasis and hyperbilirubinemia lead to chronic cholestasis or cirrhosis, often suggesting a poor prognosis. In view of this, liver biopsy is particularly important for the differential diagnosis and treatment of drug-induced cholestasis (Bessone et al., 2019). So, the treatment of cholestasis or bile duct injury is particularly important in the management of DILI. 


\section{Ursodeoxycholic Acid}

UDCA, a naturally occurring hydrophilic bile acid, which main mechanism is to reduce the cholesterol saturation index of bile and inhibit the absorption of cholesterol in intestine, has been reported to have a variety of hepatoprotective effect, such as cell protective, anti-apoptotic (Güldütuna et al., 1993), immunomodulatory (Calmus et al., 1990) and cholagogues effects (Beuers et al., 1993). It was the only FDA approved drug for treatment of primary biliary cholangitis (PBC) and has also been successfully used in various cholestatic liver diseases. Both European Association for the Study of the Liver (EASL) and Asia Pacific Association of Study of Liver (APASL) guidelines indicated that it was commonly used for DILI with cholestasis, although clinical evidence was not sufficient yet and therapeutic effect needs to be further studied (European Association for the Study of the Liver. 2019; Devarbhavi et al., 2021). A prospective pilot study investigated the hepatoprotective effectiveness of UDCA to tuberculosis- or non-tuberculosis mycobacterial-infected patients with DILI. The results revealed that all patients treated with UDCA show radiological and clinical improvement with no side effects (Lang et al., 2019). A retrospective study in China showed that UDCA can accelerate the recovery of TBIL, DBIL, Bile acid and GGT in patients with moderate and severe DILI while continuous treatment is required for more than 2 weeks (Sun et al., 2015). A clinical pilot study from Iran described pediatric patient with significant elevation ( $>2 \mathrm{ULN})$ in AST, ALT and $\gamma$-GGT) secondary to anticonvulsants confirmed at monthly follow up. The results showed a significant improvement of liver tests after treatment with UDCA for at least 24 weeks $(n=22)$ although due to uncontrolled design of this study a firm conclusion cannot be drawn (Asgarshirazi et al., 2015). However, some studies have refuted the effects of UDCA in DILI. Saito Z et al. retrospectively studied the effect of UDCA in sever DILI patients and found that UDCA did not improve normalization time or resolution rate compared with non-UDCA therapy. Further, the recovery time in UDCA treatment group was even longer (Saito et al., 2016). Additionally, UDCA has recently been recommended for druginduced VBDS with cholestasis (Bessone et al., 2021), although a few cases of inefficacy have been reported (Xu et al., 2021). Recently, a systematic review of the role of UDCA in the treatment and prevention of DILI was performed, it commented that UDCA seems to have some benefits in the treatment of DILI. However, the design of the published studies does not allow a firm conclusion to be drawn on the efficacy of UDCA in DILI. A well designed RCT to evaluate the role of UDCA in DILI is needed. (Robles-Díaz Mercedes, et al., 2021).

\section{S-Adenosylmethionine}

SAMe is the active form of methionine in the body. It is formed from substrate L-methionine and ATP catalyzed by s-adenosylmethionine synthetase. Due to its transformation of methyl, sulfur and aminopropyl groups, SAMe is involved in the detoxification of bile acid metabolism and the generation of glutathione, improves cell membrane fluidity, promotes the synthesis and secretion of bile acid in many aspects, and prevents liver cell injury and necrosis (Finkelstein, 1990; Bottiglieri et al., 1994; Lieber, 1999). As early as 1990, SAMe was summarized as an effective treatment for drug-induced intrahepatic cholestasis, including estrogen, taurolithocholate, chlorpromazine, etc. (Almasio et al., 1990). It cound relieve the symptom of pruritus and restore serum total bilirubin and serum ALP towards normal. Perlamutrov $\mathrm{Y}$ et al. conducted an multicentric, non-interventional, prospective observational study enrolled 105 patients having DILI with cholestasis (by immunosuppressives) to characterize Russian population receiving SAMe as a hepatoprotectant against DILI triggered due to immunosuppressive drugs. The results revealed that the levels of ALT, AST, TBIL, ALP, and GGT significantly decreased after SAMe treatment and the symptoms of intrahepatic cholestasis such as pruritus, fatigue and jaundice improved (Perlamutrov et al., 2014). A prospective, multicenter, observational program found that SAMe significantly reduced ALT and AST in patient with chemotherapy induced liver injury after 2 weeks treatment (Noureddin et al., 2020). Santini D et al. observed 50 cancer patients who developed anticancer chemotherapy-induced liver toxicity, and received SAMe supplementation. They found that AST, ALT and LDH decrease after one or 2 weeks of SAMe treatment. This results clearly demonstrated a protective effect of SAMe in cancer chemotherapy-induced liver toxicity (Santini et al., 2003).

\section{Cholestyramine}

Cholestyramine, also known as Calleenamine, is a nonabsorbable styrene anion exchange resin. After oral administration, it binds with intestinal cholic acid, hindering the reabsorption of cholic acid and increasing the excretion of cholic acid by $3 \sim 15$ times compared with normal. In clinical practice, cholestyramine was often used to relieve itchy skin symptoms in patients with cholestasis. As specific therapy, it was recommended by EASL and APASL guidelines that administration of cholestyramine may be used to decrease the course of hepatotoxicity induced by very selected drugs, such as leflunomide and terbinafine (European Association for the Study of the Liver. 2019; Devarbhavi et al., 2021). In the liver, leflunomide is a substrate of the hepatic microsomal enzymes CYP2C9, CYP3A4, and CYP2A1. Drugs that have an inducing or inhibitory effect on these hepatic microsomal enzymes carry the risk of adverse drug interactions with leflunomide (Alamri Raghad et al., 2021). Because the active metabolite of leflunomide is detectable in plasma until 2 years after discontinuation of the drug, cholestyramine must be given to enhance elimination from the body until plasma levels of leflunomide are undetectable ( $\varnothing$ stensen et al., 2006). Cholestyramine given orally in a dose of $8 \mathrm{~g} 3$ times daily for $24 \mathrm{~h}$ reduces plasma concentrations of leflunomide by approximately $40 \%$ in $24 \mathrm{~h}$ and $65 \%$ in $48 \mathrm{~h}$. A systematic review summarized a suggested algorithm for bile acid washout with cholestyramine for leflunomide toxicity, which can provide reference for clinical practice (Stine and Lewis, 2016). 


\section{Immunosuppressants}

Adaptive immune attack may be the final common event of DILI. Inflammatory responses are mainly a combination of immune activation and a series of related cellular and molecular events (Chalasani and Björnsson, 2010; Fontana, 2014). Intrahepatic inflammation caused by non-drug factors is an independent predisposing factor for DILI and also a factor that promotes the progression of DILI (Eisenberg-Lerner and Kimchi, 2012). Adaptive immune responses can mediate DILI and also lead to extrahepatic immune injury and then produce systemic manifestations including fever and rashes. Thus, immunosuppressants and anti-inflammatory drugs such as glucocorticoids (GCs) are also helpful in DILI treatment.

\section{Glucocorticoids}

As steroids, glucocorticoids (GCs) have been widely used in clinic practice, due to its anti-inflammatory, immunosuppressive, antiallergic and anti-shock effects. GCs was recommended for severe DILI patients in cases where the patients' serum TBIL levels are exacerbated under regular therapy, regardless of whether serum ALT level decreases or not (Hu and Xie, 2019). In addition, GCs could be empirically given to patients with marked signs of hypersensitivity or autoimmunity, and patients without remarkable improvement or even aggravation of biochemical indicators after withdrawal offending $\operatorname{drug}(\mathrm{s})$. A step-down therapy with gradually tapering off the dose of steroid seems to be used most often in clinical practice. Prednisone ranging from 15 to $20 \mathrm{mg} / \mathrm{kg} /$ day for 3 days was reported to treat successfully patients with severe DILI without obvious side effects. Abrupt withdrawal of the corticosteroids is not recommended because it may sometimes result in the rebound of liver injury (Hu and Xie, 2019). Analysis based on the American DILI Network Database showed that GCs were used in up to $82.0 \%$ of the DILI patients who died or underwent liver transplantation and $36.6 \%$ of DILI patients who survived (Fontana et al., 2014). Although the controversy about scientific evidence for efficacy of GCs has raged unabated for many years, some studies did confirm its effective role in DILI. Hou FQ and colleagues retrospectively investigated 70 patients DILI with hepatocellular injury, whose TBIL $\geq 10 \times$ ULN. The results revealed that the patients treated with GCs had higher DILI resolution rate with no side effects. Furthermore, the recovery time was longer in the patients who did no received GCs compared to those who did (Hou et al., 2012). A singlecenter retrospective study conducted by $\mathrm{Hu}$ PF et al. included 203 patients with severe DILI showed that 53 patients had a higher recovery rate and shorter recovery time after GCs treatment. They concluded that short-time use of corticosteroids is strongly recommended for severe DILI patients with hyperbilirubinemia (Hu et al., 2016). Sundaram S and Herroero-Herrero JI also respectively reported successful treatment of DILI with GCs (Herrero-Herrero and García-Aparicio, 2010; Sundaram et al., 2020). On the contrary, several investigations refuted the effects of GCs in DILI. Karkhanis J and coworkers retrospectively evaluated the effect of steroids in ALF. They collected clinical data of 131 patients with drug-induced ALF in which 16 received GCs treatment. The results revealed that GCs did not improve overall survival rate in drug-induced ALF (Karkhanis et al., 2014). Wan YM et al. enrolled 90 patients with severe DILI to investigate efficacy and safety of prednisone. The results revealed that prednisone therapy is safe but not beneficial, and even detrimental at a daily dose $>40 \mathrm{mg}$ for the treatment of severe DILI (Wan et al., 2019). Pang L and coworkers retrospectively studied the features and outcomes of hospitalized patients with DILI. They found that the recovery time and recovery rate of 32 acute DILI patients treated with GCs were not significantly improved compared with non-GCS group. However, encouragingly, the rate of resolution for patients with fulminant liver failure was superior to that of the non-steroid therapy group (57.1 vs. 25.0\%) (Pang et al., 2018). These results suggest that GCs may have a potential therapeutic effect on DILI, but inappropriate dosage and timing may be counterproductive. Thus, GCs should be fully evaluated before application and used with great caution in DILI.

\section{Specific Drugs Treatment}

In addition to hepatoprotective drugs, anticholestatic drugs, immunosuppressants, there are some specific drugs that play an important role in DILI treatment. For example, L-carnitine was used for liver injury caused by 3-keto-valproic acid (VPA). Anticoagulants such as low-molecular-weight heparins and defibrotide were used for sinusoidal obstruction syndrome (SOS)/hepatic veno-occlusive disease (VOD).

\section{L-Carnitine}

LC is a vitamin-like amino acid derivative, mainly derived from daily diet, especially red meat and dairy products. It plays a key role in physiological metabolism include promoting the transport of long-chain fatty acids, preventing the accumulation of acetylCoA in mitochondria, and thus promoting the $\beta$ oxidation of fatty acids, which further are widely used in weight-loss products (Lheureux et al., 2005). In 1982, reduced serum free carnitine as well as reduced levels of 3-keto-valproic acid (VPA), the main metabolite of $\beta$-oxidation of VPA, was first reported by Bohles and coworkers in a 3-year-old girl who developed acute liver disease with typical features of Reye's syndrome after treatment with VPA for 6 months (Böhles et al., 1982). Subsequently, Krähenbühl et al. (1995) found that the LC content of plasma and liver were reduced in a patient with fatal liver injury caused by VAP. Bohan TP and coworkers conducted a retrospective cohort study of 92 patients with VAP-induced fatal hepatotoxicity. They found that $48 \%$ survived of 42 patients treated with LC and of 50 patients who only received supportive treatment, 10\% survived (Bohan et al., 2001). Although low quality grading of evidence, EASL and APASL guidelines both recommend LC for liver injury caused by VPA.

\section{Anticoagulants}

Drug-induced liver vascular injury is relatively rare with unknown pathogenesis, in which the target cells can be hepatic sinus endothelial cells, hepatic venules, as well as the hepatic vein and the portal vein, and the clinical types include sinusoidal obstruction syndrome (SOS)/hepatic veno-occlusive disease (VOD) (Gao et al., 2012; Fan and Crawford, 2014), 
peliosis hepatis (PH) (Yu et al., 2014; Kootte et al., 2015), BuddChiari syndrome (BCS), idiopathic portal hypertension (IPH) induced by portal sclerosis, etc. Drugs that caused vascular liver injury usually included some chemotherapeutic agents such as Irinotecan or Oxaliplatin (Bai et al., 2016), herbs containing pyrrolizidine alkaloids (Leise et al., 2014), etc. There were also clinical evidences that oral contraceptives (OC) use played a role to enhance the underlying thrombophilia, due the production of clotting factor (Scarsi et al., 2016), leading the development of hepatic vein thrombosis (Perarnau and Bacq, 2008). For SOS/ VOD, supportive symptomatic treatment is the basic pyrrolidine alkaloid-related hepatic sinusoidal obstruction syndrome (PAHSOS) treatment regimen. And the early use of anticoagulants such as low-molecular-weight heparins after ruling out contraindications may have some therapeutic effect. Wang et al. summarized the long-term follow-up results of different $\mathrm{PA}-\mathrm{HSOS}$ treatment regimens in a retrospective monocenter study. 85 patients with Gynura segetum caused VOD were divided into two groups according to whether they had received anticoagulant therapy (low molecular weight heparin, warfarin) on the basis of treatment of liver function protection and microcirculation improvement. Among 22 patients who received no anticoagulant treatment, $6(27.3 \%)$ patients were cured and $14(63.6 \%)$ patients died during the treatment period. Among 63 patients treated with anticoagulant, 6 (9.5\%) patients died and 36 (57.1\%) patients were cured. The results showed that the cure rate was higher than that of non-anticoagulant treatment (Wang et al., 2016). Additionally, defibrotide is the only drug demonstrated to be effective for the prevention and treatment of hematopoietic stem cell transplantation related hepatic sinusoidal obstruction syndrome (HSCT-HSOS) (Richardson et al., 1998; Dignan et al., 2013; Richardson et al., 2016).

\section{Combination of Therapeutic Drugs}

The mixed phenotype of DILI was often associated with multiple manifestations of cholestasis and hepatocyte injury, with significantly increased all TBIL, ALP, ALT and AST. Despite the lack of adequate evidence-based medical evidence, it is not uncommon for clinicians to choose two even more hepatoprotective drugs for DILI treatment in clinical practice. A clinical study involving 40 Chinese patients with DILI during chemotherapy after radical gastritis showed that PPC combined with bicyclol significantly reduced serum ALT, AST and ALP levels compared with PPC treatment (Wu et al., 2020). A clinical study conducted by Wang HX et al. found that MgIG combined with NAC treatment significantly reduced serum malondialdehyde (MDA), interleukin-6 (IL-6) and procalcitonin (PCT), while increased the levels of glutathione peroxidase (GSH-Px) and superoxide dismutase (SOD) in DILI patients, suggesting a good anti-inflammatory and antioxidant effect (Wang et al., 2020). Qing Xie's team retrospectively analyzed the efficacy of UDCA combined with SAMe in treatment of cholestatic DILI, and found that patients received the combination therapy had a better efficacy rate than SAMe alone, with better safety (Ye et al., 2017). In a comparative analysis of clinical data from 15 patients with severe DILI, Wree A et al. found that Steroid combined with UDCA therapy appeared to be safe, and leads to a more rapid reduction in bilirubin and transaminases (Wree et al., 2011). The combination of two or more anti-inflammatory and hepatoprotective drugs has not been recommended in DILI guidelines circulated internationally, and it is urgent to carry out multi-center clinical studies with large samples to provide more adequate evidence-based medical evidence (Yue et al., 2017).

\section{NON-PHARMACOLOGICAL SUPPORT THERAPY}

Most cases of DILI are self-limiting and resolve with prompt identification and cessation of the offending agent. The patients with abnormal liver function after drug withdrawal and chronic DILI (persistent abnormalities in liver-associated enzymes at 3-6 months after drug withdrawal) (Andrade et al., 2006; Chalasani et al., 2015) are often require pharmacotherapy as described above. However, for DILI patients with severe hepatic encephalopathy, coagulation disorders or decompensated liver cirrhosis, non-pharmacological support therapy need to be considered, such as hemodialysis, peritoneal dialysis, artificial extracorporeal liver support and liver transplantation.

\section{Liver Transplantation}

Acute DILI had been reported to occur in $5-10 \%$ of patients hospitalized for jaundice (Björnsson et al., 2003), and more often evolved to fulminant hepatic failure than other causes of acute hepatic injury in Western countries (Ostapowicz et al., 2002; Wei et al., 2007). DILI is an important cause of ALF with significant morbidity and mortality. According to Russo MW et al. statistical analysis of the United Network for Organ Sharing (UNOS) liver transplant database from 1990 to 2002 , it showed that liver transplantation for drug hepatotoxicity accounted for $15 \%$ of liver transplants for ALF in US, in which mostly attributed to APAP (Russo et al., 2004). Thus, Liver transplantation should be considered for patients with ALF/SALF who present with hepatic encephalopathy and severe coagulation disorders, as well as decompensated cirrhosis (Reuben et al., 2010). All the American College of Gastroenterology (ACG), EASL and APASL clinical practice guidelines pointed out, liver transplantation was still the primary rescue treatment for ALF (European Association for the Study of the Liver. 2019; Chalasani Naga et al., 2021; Devarbhavi et al., 2021), with 1 and 5 years survival benefits of about $70-80 \%$ in liver transplants for DILIALF (Adam et al., 2018).

\section{Artificial Extracorporeal Liver Support}

By means of in vitro mechanical, chemical or biological devices, AELS is a temporary or partial replacement of liver function to assist in the treatment of liver dysfunction or related diseases, mainly including biological artificial liver (BAL), no-biological artificial liver (NBAL) and their mixture. NBAL plays an important role in clinical practice, but its curative effect remains to be discussed. An open randomized controlled trial in regard to High-volume plasma exchange (HVP) in patients 
with ALF showed that treatment with HVP can increase liver transplant-free survival ( 58.7 vs. $47.8 \%$ ), and the incidence of severe adverse events was similar to control group (Larsen et al., 2016). But, another trial about extracorporeal albumin dialysis with the molecular adsorbent recirculating system in acute-onchronic liver failure revealed that the beneficial effect of it cannot be verified (Bañares et al., 2013). A review also concluded that the sole use of devices such as hemodialysis and albumin dialysis aimed to remove toxins is not sufficient to improve survival in patients with ALF (Larsen, 2019).

\section{CONCLUSION AND FUTURE PERSPECTIVES}

DILI is one of the most common and serious adverse drug reactions (ADRs), leading to acute liver failure (ALF) and even death in severe cases (Li et al., 2007; Miguel et al., 2012; Björnsson et al., 2013). Up to now, there is still lack of simple, objective and specific diagnostic indexes and treatment methods due to its complex pathogenesis. Thus, it is vital to identify the offending $\operatorname{drug}(\mathrm{s})$ and prompt withdrawal before irreversible liver damage. The DILI Collaboration Network (DILIN) was founded in the United States in 2003, and a prospective study of DILIN (DILIN-PS) was initiated next year (Fontana et al., 2009). The LiverTox website (http:// www.livertox.nih.gov) set up by DILIN in 2012 and HepaTox website (http://www.hepatox.org) by Chinese Medical Association drug-induced liver disease Group in 2014 records information on more than 1,000 common drugs for liver damage. It can provide a basis for avoiding the use of potential hepatotoxic drugs in clinical practice.

For pharmacological therapy of DILI, there is a lack of normative guidance or guidelines. There is usually a complete or near complete resolution of DILI within a matter of days to weeks after offending drug withdrawal. The starting point of protective drugs intervention is still difficult to choose. Therefore, dynamic monitoring of liver biochemical parameters is recommended by all clinical practice guidelines. Based on the type of target cells damaged, patients tend to show different clinical phenotypes of DILI with a distinction in liver biochemical indicators. In this paper, we reviewed a large amount of evidencebased medical studies and summarized the commonly used drugs for DILI in clinical practice. NAC, MgIG, PPC, bicyclol, silymarin are potential hepatoprotective drugs for DILI. In anticholestatic drugs, UDCA, SAMe, cholestyramine showed therapeutic value for DILI. LC is recommended for liver injury caused by VPA. GCs is suitable for patients with obvious hypersensitivity or autoimmune symptoms and no obvious improvement in liver biochemical indicators after withdrawal. Anticoagulants, such as low-molecular-weight heparins and defibrotide, play irreplaceable roles in some special types of DILI (eg. PAHSOS, etc.). They could provide reference for the selection and combined application of protective drugs for different clinical phenotypes of DILI. Nevertheless, the definite therapeutic effects of these drugs are still to be confirmed by prospective randomized and controlled studies.

The onset of DILI is a complex process, and its pathogenesis has become a research hotspot in recent years. With further research into the pathological mechanisms and the rapid development of techniques, many new therapeutic drugs may shed light on DILI clinical management. Emerging therapeutic interventions for DILI include gene therapy, nano-therapy, structural modification of toxic drugs, etc. For example, the role of $\mathrm{C}-\mathrm{X}-\mathrm{C}$ motif chemokine ligand-9 (CXCL9) in APAP-induced liver injury was reported crucial recently. Despite the lack of clinical trials, hepatocyte apoptosis of APAP-treated mice decreased following administration of a CXCL9 neutralizing antibody or CXCR3 gene knockout (Song et al., 2019). In humans, genetically inherited defects in BSEP expression or activity cause cholestatic liver injury, and many drugs that cause cholestatic drug-induced liver injury (DILI) in humans have been shown to inhibit BSEP activity in vitro and in vivo (Saran et al., 2021). Attempts at molecular structural modification of hepatotoxic drugs that inhibit BSEP may provide new strategies for treatment of cholestatic DILI (Warner et al., 2012). Exciting work in the field of nanotechnology holds the promise of developing novel treatment and prevention approaches for DILI in the not-too-distant future. The ability to deliver inhibitors of inflammatory cytokines, apoptosis and other cellular events leading to liver cell necrosis and death directly to hepatocytes or other substructures to prevent DILI is an attractive possibility for the future, and is being actively pursued (Isoda et al., 2011; Reddy and Couvreur, 2011; Cui et al., 2012; Momen-Heravi et al., 2014).

\section{AUTHOR CONTRIBUTIONS}

ML and XS wrote the manuscript and prepared the tables; QL collected the literatures; YT, XS, and CL contributed to writing and reviewing the manuscript. All authors approved the final version of the manuscript.

\section{FUNDING}

This work was supported by three research program grants: Shanghai TCM development three-year action plan project (ZY-(2018-2020)-CCCX-5001), Shanghai Science and Technology Innovation Action Plan medical innovation research special project (20Z21900100) and Shanghai Key Specialty of Traditional Chinese Clinical Medicine (shslczdzk01201). 


\section{REFERENCES}

Adam, R., Karam, V., Cailliez, V., O Grady, J. G., Mirza, D., Cherqui, D., et al. (2018). 2018 Annual Report of the European Liver Transplant Registry (ELTR)50-year Evolution of Liver Transplantation. Transpl. Int. 31 (12), 1293-1317. doi:10.1111/tri.13358

Aithal, G. P., Watkins, P. B., Andrade, R. J., Larrey, D., Molokhia, M., Takikawa, H., et al. (2011). Case Definition and Phenotype Standardization in Drug-Induced Liver Injury. Clin. Pharmacol. Ther. 89 (6), 806-815. doi:10.1038/clpt.2011.58

Aithal, P. G., and Day, C. P. (1999). The Natural History of Histologically Proved Drug Induced Liver Disease. Gut 44 (5), 731-735. doi:10.1136/gut.44.5.731

Alamri, R. D., Elmeligy, M. A., Albalawi, G. A., Alquayr, S. M., Alsubhi, S. S., and El-Ghaiesh, S. H. (2021). Leflunomide an Immunomodulator with Antineoplastic and Antiviral Potentials but Drug-Induced Liver Injury: A Comprehensive Review. Int. Immunopharmacol 93 (undefined), 107398. doi:10.1016/j.intimp.2021.107398

Almasio, P., Bortolini, M., Pagliaro, L., and Coltorti, M. (1990). Role of S-AdenosylL-Methionine in the Treatment of Intrahepatic Cholestasis. Drugs 40 (Suppl. 3), 111-123. doi:10.2165/00003495-199000403-00011

Andrade, R. J., Lucena, M. I., Kaplowitz, N., García-Muņoz, B., Borraz, Y., Pachkoria, K., et al. (2006). Outcome of Acute Idiosyncratic Drug-Induced Liver Injury: Long-Term Follow-Up in a Hepatotoxicity Registry. Hepatology 44 (6), 1581-1588. doi:10.1002/hep.21424

Asgarshirazi, M., Shariat, M., Dalili, H., and Keihanidoost, Z. (2015). Ursodeoxycholic Acid Can Improve Liver Transaminase Quantities in Children with Anticonvulsant Drugs Hepatotoxicity: a Pilot Study. Acta Med. Iran 53 (6), 351-355. doi:10.1002/hep.25531

Bai, P., Ye, H., Xie, M., Saxena, P., Zulewski, H., Charpin-El Hamri, G., et al. (2016). A Synthetic Biology-Based Device Prevents Liver Injury in Mice. J. Hepatol. 65 (1), 84-94. doi:10.1016/j.jhep.2016.03.020

Bañares, R., Nevens, F., Larsen, F. S., Jalan, R., Albillos, A., Dollinger, M., et al. (2013). Extracorporeal Albumin Dialysis with the Molecular Adsorbent Recirculating System in Acute-On-Chronic Liver Failure: the RELIEF Trial. Hepatology 57 (3), 1153-1162. doi:10.1002/hep.26185

Bessone, F., Hernández, N., Tanno, M., and Roma, M. G. (2021). Drug-Induced Vanishing Bile Duct Syndrome: From Pathogenesis to Diagnosis and Therapeutics. Semin. Liver Dis. 41 (3), 331-348. doi:10.1055/s-0041-1729972

Bessone, F., Robles-Diaz, M., Hernandez, N., Medina-Caliz, I., Lucena, M. I., and Andrade, R. J. (2019). Assessment of Serious Acute and Chronic Idiosyncratic Drug-Induced Liver Injury in Clinical Practice. Semin. Liver Dis. 39 (3), 381-394. doi:10.1055/s-0039-1685519

Beuers, U., Nathanson, M. H., Isales, C. M., and Boyer, J. L. (1993). Tauroursodeoxycholic Acid Stimulates Hepatocellular Exocytosis and Mobilizes Extracellular Ca++ Mechanisms Defective in Cholestasis. J. Clin. Invest. 92 (6), 2984-2993. doi:10.1172/JCI116921

Björnsson, E., Ismael, S., Nejdet, S., and Kilander, A. (2003). Severe Jaundice in Sweden in the New Millennium: Causes, Investigations, Treatment and Prognosis. Scand. J. Gastroenterol. 38 (1), 86-94. doi:10.1080/ 00365520310000492

Björnsson, E., and Olsson, R. (2005). Outcome and Prognostic Markers in Severe Drug-Induced Liver Disease. Hepatology 42 (2), 481-489. doi:10.1002/ hep. 20800

Björnsson, E. S., Bergmann, O. M., Björnsson, H. K., Kvaran, R. B., and Olafsson, S. (2013). Incidence, Presentation, and Outcomes in Patients with Drug-Induced Liver Injury in the General Population of Iceland. Gastroenterology 144 (7), 1419-1425. doi:10.1053/j.gastro.2013.02.006

Bohan, A., and Boyer, J. L. (2002). Mechanisms of Hepatic Transport of Drugs: Implications for Cholestatic Drug Reactions. Semin. Liver Dis. 22 (2), 123-136. doi:10.1055/s-2002-30099

Bohan, T. P., Helton, E., McDonald, I., König, S., Gazitt, S., Sugimoto, T., et al. (2001). Effect of L-Carnitine Treatment for Valproate-Induced Hepatotoxicity. Neurology 56 (10), 1405-1409. doi:10.1212/wnl.56.10.1405

Böhles, H., Richter, K., Wagner-Thiessen, E., and Schäfer, H. (1982). Decreased Serum Carnitine in Valproate Induced Reye Syndrome. Eur. J. Pediatr. 139 (3), 185-186. doi:10.1007/BF01377353

Borah, A., Paul, R., Choudhury, S., Choudhury, A., Bhuyan, B., Das Talukdar, A., et al. (2013). Neuroprotective Potential of Silymarin against CNS Disorders:
Insight into the Pathways and Molecular Mechanisms of Action. CNS Neurosci. Ther. 19 (11), 847-853. doi:10.1111/cns.12175

Borlak, J., van Bömmel, F., and Berg, T. (2018). N-acetylcysteine and Prednisolone Treatment Improved Serum Biochemistries in Suspected Flupirtine Cases of Severe Idiosyncratic Liver Injury. Liver Int. 38 (2), 365-376. doi:10.1111/ liv.13538

Bottiglieri, T., Hyland, K., and Reynolds, E. H. (1994). The Clinical Potential of Ademetionine (S-Adenosylmethionine) in Neurological Disorders. Drugs 48 (2), 137-152. doi:10.2165/00003495-199448020-00002

Calmus, Y., Gane, P., Rouger, P., and Poupon, R. (1990). Hepatic Expression of Class I and Class II Major Histocompatibility Complex Molecules in Primary Biliary Cirrhosis: Effect of Ursodeoxycholic Acid. Hepatology 11 (1), 12-15. doi:10.1002/hep.1840110104

Chalasani, N., and Björnsson, E. (2010). Risk Factors for Idiosyncratic DrugInduced Liver Injury. Gastroenterology 138 (7), 2246-2259. doi:10.1053/ j.gastro.2010.04.001

Chalasani, N., Bonkovsky, H. L., Fontana, R., Lee, W., Stolz, A., Talwalkar, J., et al. (2015). United States Drug Induced Liver Injury NetworkFeatures and Outcomes of 899 Patients with Drug-Induced Liver Injury: The DILIN Prospective Study. Gastroenterology 148 (7), 1340-1352. e7. doi:10.1053/ j.gastro.2015.03.006

Chalasani, N. P., Maddur, H., Russo, M. W., Wong, R. J., and Reddy, K. R. (2021). Practice Parameters Committee of the American College of GastroenterologyACG Clinical Guideline: Diagnosis and Management of Idiosyncratic Drug-Induced Liver Injury. Am. J. Gastroenterol. 116 (5), 878-898. doi:10.14309/ajg.0000000000001259

Chen, D. F., and Sun, W. J. (2011). The Application of the Anti-inflammatory and Hepatoprotective Drugs on Drug-Induced Liver Disease. Chin. J. Hepatol. 1 (03), 232-233. doi:10.3760/cma.j.issn.1007-3418.2011.03.023

Chen, Y., Ye, P., Ren, C., Ren, P., Ma, Z., Zhang, L., et al. (2018). Pharmacoeconomics of Three Therapeutic Schemes for Anti-tuberculosis Therapy Induced Liver Injury in China. Open Med. (Wars) 13, 53-63. doi:10.1515/med-2018-0010

Chiew, A. L., Gluud, C., Brok, J., and Buckley, N. A. (2018). Interventions for Paracetamol (Acetaminophen) Overdose. Cochrane Database Syst. Rev. 2 (2), CD003328. doi:10.1002/14651858.CD003328.pub3

Chinese Society of Infectious Diseases (2014). Consensus Statement by the Expert Committee for Prevention and Management of Fiver Inflammation in China. Chin. J. Hepato 22 (02), 94-103. doi:10.3760/cma.j.issn.1007-3418.2014.02.006 Committee of experts on silymarin therapy in patients with liver disease (2016). Experts Consensus on Silymarin Therapy in Patients with Liver Diseases. Chin. J. Exp. Clin. Infect. Dis. (Electronic Edition) 10 (05), 517-521. doi:10.3877/ cma.j.issn.1674-1358.2016.05.002

Committee of the treatment with polyenphosphatidylcholine in patients with liver diseases (2017). Experts Consensus on the Treatment with Polyenphosphatidylcholine in Patients with Liver Diseases. Chin. J. Exp. Clin. Infect. Dis. (Electronic Edition) 11 (04), 313-319. doi:10.3877/ cma.j.issn.1674-1358.2017.04.001

Cui, Y., Liu, H., Ze, Y., Zengli, Z., Hu, Y., Cheng, Z., et al. (2012). Gene Expression in Liver Injury Caused by Long-Term Exposure to Titanium Dioxide Nanoparticles in Mice. Toxicol. Sci. 128 (1), 171-185. doi:10.1093/toxsci/kfs153

Dekhuijzen, P. N. (2004). Antioxidant Properties of N-Acetylcysteine: Their Relevance in Relation to Chronic Obstructive Pulmonary Disease. Eur. Respir. J. 23 (4), 629-636. doi:10.1183/09031936.04.00016804

Devarbhavi, H., Aithal, G., Treeprasertsuk, S., Takikawa, H., Mao, Y., Shasthry, S. M., et al. (2021). Drug-induced Liver Injury: Asia Pacific Association of Study of Liver Consensus Guidelines. Hepatol. Int. 15 (2), 258-282. doi:10.1007/s12072021-10144-3

Devarbhavi, H., Dierkhising, R., Kremers, W. K., Sandeep, M. S., Karanth, D., and Adarsh, C. K. (2010). Single-center Experience with Drug-Induced Liver Injury from India: Causes, Outcome, Prognosis, and Predictors of Mortality. Am. J. Gastroenterol. 105 (11), 2396-2404. doi:10.1038/ajg.2010.287

Dignan, F. L., Wynn, R. F., Hadzic, N., Karani, J., Quaglia, A., Pagliuca, A., et al. (2013). Haemato-oncology Task Force of British Committee for Standards in Haematology, British Society for Blood and Marrow TransplantationBCSH/ BSBMT Guideline: Diagnosis and Management of Veno-Occlusive Disease (Sinusoidal Obstruction Syndrome) Following Haematopoietic Stem Cell Transplantation. Br. J. Haematol. 163 (4), 444-457. doi:10.1111/bjh.12558 
Eisenberg-Lerner, A., and Kimchi, A. (2012). PKD Is a Kinase of Vps34 that Mediates ROS-Induced Autophagy Downstream of DAPk. Cell Death Differ 19 (5), 788-797. doi:10.1038/cdd.2011.149

Elliott, T. R., Symes, T., Kannourakis, G., and Angus, P. (2016). Resolution of Norfloxacin-Induced Acute Liver Failure afterN-Acetylcysteine Therapy: Further Support for the Use of NAC in Drug-Induced ALF? BMJ case Rep. 2016, bcr2015213189. doi:10.1136/bcr-2015-213189

Enjalbert, F., Rapior, S., Nouguier-Soulé, J., Guillon, S., Amouroux, N., and Cabot, C. (2002). Treatment of Amatoxin Poisoning: 20-year Retrospective Analysis. J. Toxicol. Clin. Toxicol. 40 (6), 715-757. doi:10.1081/clt-120014646

European Association for the Study of the Liver (2019). EASL Clinical Practice Guidelines: Drug-Induced Liver Injury. J. Hepatol. 70 (6), 1222-1261. doi:10.1016/j.jhep.2019.02.014

Expert Committee on Clinical Application of Bicyclol (2020). Expert Consensus on Clinical Application of Bicyclol--2020 Edition. Chin. J. Exp. Clin. Infect. Dis. (Electronic Edition) 14 (03), 177-185. doi:10.3877/cma.j.issn.16741358.2020.03.001

Fan, C. Q., and Crawford, J. M. (2014). Sinusoidal Obstruction Syndrome (Hepatic Veno-Occlusive Disease). J. Clin. Exp. Hepatol. 4 (4), 332-346. doi:10.1016/ j.jceh.2014.10.002

Finkelstein, J. D. (1990). Methionine Metabolism in Mammals. J. Nutr. Biochem. 1 (5), 228-237. doi:10.1016/0955-2863(90)90070-2

Fontana, R. J., Hayashi, P. H., Gu, J., Reddy, K. R., Barnhart, H., Watkins, P. B., et al. (2014). Idiosyncratic Drug-Induced Liver Injury Is Associated with Substantial Morbidity and Mortality within 6 Months from Onset. Gastroenterology 147 (1), 96-e4. doi:10.1053/j.gastro.2014.03.045

Fontana, R. J. (2014). Pathogenesis of Idiosyncratic Drug-Induced Liver Injury and Clinical Perspectives. Gastroenterology 146 (4), 914-928. doi:10.1053/ j.gastro.2013.12.032

Fontana, R. J., Watkins, P. B., Bonkovsky, H. L., Chalasani, N., Davern, T., Serrano, J., et al. (2009). Drug-Induced Liver Injury Network (DILIN) Prospective Study: Rationale, Design and Conduct. Drug Saf. 32 (1), 55-68. doi:10.2165/00002018200932010-00005

Gao, H., Li, N., Wang, J. Y., Zhang, S. C., and Lin, G. (2012). Definitive Diagnosis of Hepatic Sinusoidal Obstruction Syndrome Induced by Pyrrolizidine Alkaloids. J. Dig. Dis. 13 (1), 33-39. doi:10.1111/j.1751-2980.2011.00552.x

Grabhorn, E., Nielsen, D., Hillebrand, G., Brinkert, F., Herden, U., Fischer, L., et al. (2013). Successful Outcome of Severe Amanita Phalloides Poisoning in Children. Pediatr. Transpl. 17 (6), 550-555. doi:10.1111/petr.12108

Güldütuna, S., Zimmer, G., Imhof, M., Bhatti, S., You, T., and Leuschner, U. (1993). Molecular Aspects of Membrane Stabilization by Ursodeoxycholate [see Comment]. Gastroenterology 104 (6), 1736-1744. doi:10.1016/0016-5085(93) 90653-t

Hayashi, P. H., and Fontana, R. J. (2014). Clinical Features, Diagnosis, and Natural History of Drug-Induced Liver Injury. Semin. Liver Dis. 34 (2), 134-144. doi:10.1055/s-0034-1375955

Herrero-Herrero, J. I., and García-Aparicio, J. (2010). Corticosteroid Therapy in a Case of Severe Cholestasic Hepatitis Associated with Amoxicillin-Clavulanate. J. Med. Toxicol. 6 (4), 420-423. doi:10.1007/s13181-010-0019-4

Hou, F. Q., Zeng, Z., and Wang, G. Q. (2012). Hospital Admissions for DrugInduced Liver Injury: Clinical Features, Therapy, and Outcomes. Cell Biochem Biophys 64 (2), 77-83. doi:10.1007/s12013-012-9373-y

Hu, P. F., Wang, P. Q., Chen, H., Hu, X. F., Xie, Q. P., Shi, J., et al. (2016). Beneficial Effect of Corticosteroids for Patients with Severe Drug-Induced Liver Injury. J. Dig. Dis. 17 (9), 618-627. doi:10.1111/1751-2980.12383

Hu, P. F., and Xie, W. F. (2019). Corticosteroid Therapy in Drug-Induced Liver Injury: Pros and Cons. J. Dig. Dis. 20 (3), 122-126. doi:10.1111/17512980.12697

Isoda, K., Hasezaki, T., Kondoh, M., Tsutsumi, Y., and Yagi, K. (2011). Effect of Surface Charge on Nano-Sized Silica Particles-Induced Liver Injury. Pharmazie 66 (4), 278-281. doi:10.1691/ph.2011.0808

Karkhanis, J., Verna, E. C., Chang, M. S., Stravitz, R. T., Schilsky, M., Lee, W. M., et al. (2014). Acute Liver Failure Study GroupSteroid Use in Acute Liver Failure. Hepatology 59 (2), 612-621. doi:10.1002/hep.26678

Kootte, A. M., Siegel, A. M., and Koorenhof, M. (2015). Generalised Peliosis Hepatis Mimicking Metastases after Long-Term Use of Oral Contraceptives. Neth. J. Med. 73 (1), 41-43.
Krähenbühl, S., Mang, G., Kupferschmidt, H., Meier, P. J., and Krause, M. (1995). Plasma and Hepatic Carnitine and Coenzyme A Pools in a Patient with Fatal, Valproate Induced Hepatotoxicity. Gut 37 (1), 140-143. doi:10.1136/ gut.37.1.140

Lang, S. M., Ortmann, J., Rostig, S., and Schiffl, H. (2019). Ursodeoxycholic Acid Attenuates Hepatotoxicity of Multidrug Treatment of Mycobacterial Infections: A Prospective Pilot Study. Int. J. Mycobacteriol 8 (1), 89-92. doi:10.4103/ ijmy.ijmy_159_18

Larsen, F. S. (2019). Artificial Liver Support in Acute and Acute-On-Chronic Liver Failure. Curr. Opin. Crit. Care 25 (2), 187-191. doi:10.1097/ MCC. 0000000000000584

Larsen, F. S., Schmidt, L. E., Bernsmeier, C., Rasmussen, A., Isoniemi, H., Patel, V. C., et al. (2016). High-volume Plasma Exchange in Patients with Acute Liver Failure: An Open Randomised Controlled Trial. J. Hepatol. 64 (1), 69-78. doi:10.1016/j.jhep.2015.08.018

Lee, W. M., Hynan, L. S., Rossaro, L., Fontana, R. J., Stravitz, R. T., Larson, A. M., et al. (2009). Intravenous N-Acetylcysteine Improves Transplant-free Survival in Early Stage Non-acetaminophen Acute Liver Failure. Gastroenterology 137 (3), 856-e1. doi:10.1053/j.gastro.2009.06.006

Lei, X., Zhang, J., Xu, Q., Li, J., Qian, Y., Zhang, J., et al. (2021). Exploring the Efficacy and Safety of Polyene Phosphatidylcholine for Treatment of DrugInduced Liver Injury Using the Roussel Uclaf Causality Assessment Method: a Propensity Score Matching Comparison. J. Int. Med. Res. 49 (8), 3000605211039810. doi:10.1177/03000605211039810

Leise, M. D., Poterucha, J. J., and Talwalkar, J. A. (2014). Drug-induced Liver Injury. Mayo Clin. Proc. 89 (1), 95-106. doi:10.1016/j.mayocp.2013.09.016

Lheureux, P. E., Penaloza, A., Zahir, S., and Gris, M. (2005). Science Review: Carnitine in the Treatment of Valproic Acid-Induced Toxicity - what Is the Evidence? Crit. Care 9 (5), 431-440. doi:10.1186/cc3742

Li, F., Zhao, Y., Li, D., and Hu, R. (2018). Inhibition Effect of Bicyclol on Lipid Peroxidation in Liver Injury Induced by Neuropathic Drugs. Med. J. West. China 30 (03), 446-448. doi:10.3969/j.issn.1672-3511.2018.03.029

Li, L., Jiang, W., and Wang, J. (2007). Clinical Analysis of 275 Cases of Acute DrugInduced Liver Disease. Front. Med. China 1 (1), 58-61. doi:10.1007/s11684007-0012-8

Lieber, C. S. (1999). Role of S-Adenosyl-L-Methionine in the Treatment of Liver Diseases. J. Hepatol. 30 (6), 1155-1159. doi:10.1016/s0168-8278(99)80274-8

Liu, X., Zhao, M., Mi, J., Chen, H., Sheng, L., and Li, Y. (2017). Protective Effect of Bicyclol on Anti-tuberculosis Drug Induced Liver Injury in Rats. Molecules 22 (4), 524. doi:10.3390/molecules22040524

Liu, Y. H., Da, R. F., Xu, H. B., Jiang, Y., and Xie, H. P. (2021). Therapeutic Efficacy of Polyene Phosphatidylcholine in Patients with Anti-tuberculosis AgentsInduced Liver Injury. J. Prac Hepatol. 24 (02), 228-231. doi:10.3969/j.issn.16725069.2021.02.020

Mao, Y. M., Zeng, M. D., Chen, Y., Chen, C. W., Fu, Q. C., Cai, X., et al. (2009). Magnesium Isoglycyrrhizinate in the Treatment of Chronic Liver Diseases: a Randomized, Double-Blind, Multi-Doses, Active Drug Controlled, Multicenter Study. Randomized Controlled Trial 17 (11), 847-851. doi:10.3760/ cma.j.issn.1007-3418.2009.11.01310.1364/oe.17.008641

Marjani, M., Fahim, F., Sadr, M., Kazempour Dizaji, M., Moniri, A., Khabiri, S., et al. (2019). Evaluation of Silymarin for Management of Anti-tuberculosis Drug Induced Liver Injury: a Randomized Clinical Trial. Gastroenterol. Hepatol. Bed Bench 12 (2), 138-142. doi:10.22037/ghfbb.v12i2.1562

Masoumeh, A., Mamak, S., and Sabereh, M. (2015). Comparison between Ursodeoxycholic Acid and Silymarin in Anticonvulsive Drugs Induced Hypertransaminasemia. Inflamm. Cel Signal 2, e971. doi:10.14800/ics.971

Miguel, A., Azevedo, L. F., Araújo, M., and Pereira, A. C. (2012). Frequency of Adverse Drug Reactions in Hospitalized Patients: a Systematic Review and Meta-Analysis. Pharmacoepidemiol. Drug Saf. 21 (11), 1139-1154. doi:10.1002/ pds.3309

Momen-Heravi, F., Bala, S., Bukong, T., and Szabo, G. (2014). Exosome-mediated Delivery of Functionally Active miRNA-155 Inhibitor to Macrophages. Nanomedicine 10 (7), 1517-1527. doi:10.1016/j.nano.2014.03.014

Moosa, M. S., Maartens, G., Gunter, H., Allie, S., Chughlay, M. F., Setshedi, M., et al. (2020). A Randomized Controlled Trial of Intravenous N-Acetylcysteine in the Management of Anti-tuberculosis Drug-Induced Liver Injury. Randomized Controlled Trial 73, e3377-e3383. doi:10.1093/cid/ciaa1255 
Naiqiong, W., Liansheng, W., Zhanying, H., Yuanlin, G., Chenggang, Z., Ying, G., et al. (2017). A Multicenter and Randomized Controlled Trial of Bicyclol in the Treatment of Statin-Induced Liver Injury. Med. Sci. Monit. 23, 5760-5766. doi:10.12659/msm.904090

Noureddin, M., Sander-Struckmeier, S., and Mato, J. M. (2020). Early Treatment Efficacy of S-Adenosylmethionine in Patients with Intrahepatic Cholestasis: A Systematic Review. World J. Hepatol. 12 (2), 46-63. doi:10.4254/wjh.v12.i2.46

Ostapowicz, G., Fontana, R. J., Schiødt, F. V., Larson, A., Davern, T. J., Han, S. H., et al. (2002). Results of a Prospective Study of Acute Liver Failure at 17 Tertiary Care Centers in the United States. Ann. Intern. Med. 137 (12), 947-954. doi:10.7326/0003-4819-137-12-200212170-00007

Østensen, M., Khamashta, M., Lockshin, M., Parke, A., Brucato, A., Carp, H., et al. (2006). Anti-inflammatory and Immunosuppressive Drugs and Reproduction. Arthritis Res. Ther. 8 (3), 209. doi:10.1186/ar1957

Padda, M. S., Sanchez, M., Akhtar, A. J., and Boyer, J. L. (2011). Drug-induced Cholestasis. Hepatology 53 (4), 1377-1387. doi:10.1002/hep.24229

Pang, L., Yang, W., and Hou, F. (2018). Features and Outcomes from a Retrospective Study of 570 Hospitalized Chinese Patients with DrugInduced Liver Injury. Clin. Res. Hepatol. Gastroenterol. 42 (1), 48-56. doi:10.1016/j.clinre.2017.08.003

Patel, T., Tarun, T., Hudhud, D., and Krvavac, A. (2019). Novel Use of N-Acetylcysteine in Management of Tyrosine Kinase Inhibitor Induced Acute Liver Injury. Cureus 11 (11), e6251. doi:10.7759/cureus.6251

Perarnau, J. M., and Bacq, Y. (2008). Hepatic Vascular Involvement Related to Pregnancy, Oral Contraceptives, and Estrogen Replacement Therapy. Semin. Liver Dis. 28 (3), 315-327. doi:10.1055/s-0028-1085099

Perlamutrov, Y., Bakulev, A., Korsunskaya, I., Orlov, E., and Bolotnikova, N. (20142014). Ademetionine in Treatment of Drug Induced Liver Injury: an Observational Study in Russian Patients, Receiving Immunosuppressive Therapy for Psoriasis. IJPSR 5, 5163-5169. doi:10.13040/IJPSR.09758232.5(12).5163-69

Prescott, L. F., Illingworth, R. N., Critchley, J. A., Stewart, M. J., Adam, R. D., and Proudfoot, A. T. (1979). Intravenous N-Acetylcystine: the Treatment of Choice for Paracetamol Poisoning. Br. Med. J. 2 (6198), 1097-1100. doi:10.1136/ bmj.2.6198.1097

Reddy, L. H., and Couvreur, P. (2011). Nanotechnology for Therapy and Imaging of Liver Diseases. J. Hepatol. 55 (6), 1461-1466. doi:10.1016/j.jhep.2011.05.039

Reuben, A., Koch, D. G., and Lee, W. M. (2010). Acute Liver Failure Study GroupDrug-Induced Acute Liver Failure: Results of a U.S. Multicenter, Prospective Study. Hepatology 52 (6), 2065-2076. doi:10.1002/hep.23937

Richardson, P. G., Elias, A. D., Krishnan, A., Wheeler, C., Nath, R., Hoppensteadt, D., et al. (1998). Treatment of Severe Veno-Occlusive Disease with Defibrotide: Compassionate Use Results in Response without Significant Toxicity in a HighRisk Population. Blood 92 (3), 737-744. doi:10.1182/blood.V92.3.737

Richardson, P. G., Riches, M. L., Kernan, N. A., Brochstein, J. A., Mineishi, S., Termuhlen, A. M., et al. (2016). Phase 3 Trial of Defibrotide for the Treatment of Severe Veno-Occlusive Disease and Multi-Organ Failure. Blood 127 (13), 1656-1665. doi:10.1182/blood-2015-10-676924

Robles-Díaz, M., Nezic, L., Vujic-Aleksic, V., and Björnsson, E. S. (2021). Role of Ursodeoxycholic Acid in Treating and Preventing Idiosyncratic Drug-Induced Liver Injury. A Systematic Review. Front. Pharmacol. 12 (undefined), 744488. doi:10.3389/fphar.2021.744488

Russo, M. W., Galanko, J. A., Shrestha, R., Fried, M. W., and Watkins, P. (2004). Liver Transplantation for Acute Liver Failure from Drug Induced Liver Injury in the United States. Liver Transpl. 10 (8), 1018-1023. doi:10.1002/lt.20204

Saito, Z., Kaneko, Y., Kinoshita, A., Kurita, Y., Odashima, K., Horikiri, T., et al. (2016). Effectiveness of Hepatoprotective Drugs for Anti-tuberculosis Drug-Induced Hepatotoxicity: a Retrospective Analysis. BMC Infect. Dis. 16 (1), 668. doi:10.1186/s12879-016-2000-6

Santini, D., Vincenzi, B., Massacesi, C., Picardi, A., Gentilucci, U. V., Esposito, V., et al. (2003). S-adenosylmethionine (AdoMet) Supplementation for Treatment of Chemotherapy-Induced Liver Injury. Anticancer Res. 23 (6D), 5173-5179. doi:10.1517/14740338.2011.562888

Saran, C., Sundqvist, L., Ho, H., Niskanen, J., Honkakoski, P., and Brouwer, K. L. (2021). Novel Bile Acid-dependent Mechanisms of Hepatotoxicity Associated with Tyrosine Kinase Inhibitors. J. Pharmacol. Exp. Ther. 380, 1. doi:10.1124/jpet.121.000828
Scarsi, K. K., Darin, K. M., Chappell, C. A., Nitz, S. M., and Lamorde, M. (2016). Drug-Drug Interactions, Effectiveness, and Safety of Hormonal Contraceptives in Women Living with HIV. Drug Saf. 39 (11), 1053-1072. doi:10.1007/s40264016-0452-7

Song, X., Shen, Y., Lao, Y., Tao, Z., Zeng, J., Wang, J., et al. (2019). CXCL9 Regulates Acetaminophen-Induced Liver Injury via CXCR3. Exp. Ther. Med. 18 (6), 4845-4851. doi:10.3892/etm.2019.8122

Squires, R. H., Dhawan, A., Alonso, E., Narkewicz, M. R., Shneider, B. L., Rodriguez-Baez, N., et al. (2013). Intravenous N-Acetylcysteine in Pediatric Patients with Nonacetaminophen Acute Liver Failure: a Placebo-Controlled Clinical Trial. Hepatology 57 (4), 1542-1549. doi:10.1002/hep.26001

Stine, J. G., and Lewis, J. H. (2016). Current and Future Directions in the Treatment and Prevention of Drug-Induced Liver Injury: a Systematic Review. Expert Rev. Gastroenterol. Hepatol. 10 (4), 517-536. doi:10.1586/17474124.2016.1127756

Sun, S. S., Wu, Y. X., Wang, X. J., Ni, L. D., Jiang, T., Jin, Y. P., et al. (2015). The Efficacy of Ursodeoxycholic Acid Therapy on Moderate or Severe DrugInduced Liver Injury. Chin. Hepatol. 20 (02), 101-105. doi:10.14000/ j.cnki.issn.1008-1704.2015.02.002

Sundaram, S., Vuppalanchi, R., Saxena, R., and Chalasani, N. (2020). Treatment of Idiosyncratic Drug-Induced Liver Injury Using Steroids. ACG Case Rep. J. 7 (2), e00319. doi:10.14309/cri.0000000000000319

Tang, L. N., Lin, F., Shen, Z., Sun, Y. J., and Yao, Y. (2012). Magnesium Isoglycyrrhizinate Used in the Treatment of Chemotherapeutic DrugsInduced Acute Liver Dysfunction: A Phase III Clinical Trial. TUMOR 32 (09), 738-743. doi:10.3781/j.issn.1000-7431.2012.09.012

Tittel, G., and Wagner, H. (1977). [High-performance Liquid Chromatographic Separation of Silymarins and Their Determination in a Raw Extract of Silybum marianum Gaertn]. J. Chromatogr. 135 (2), 499-501. doi:10.1016/s0021-9673(00)88397-1

Wan, Y. M., Wu, J. F., Li, Y. H., Wu, H. M., Wu, X. N., and Xu, Y. (2019). Prednisone Is Not Beneficial for the Treatment of Severe Drug-Induced Liver Injury: An Observational Study (STROBE Compliant). Medicine (Baltimore) 98 (26), e15886. doi:10.1097/MD.0000000000015886

Wang, H. X., Zhu, Q. J., Zhang, Y. J., and Mao, C. (2020). Efficacy of Acetylcysteine Combined with Magnesium Isoglycyrrhizinate in Treatment of Patients with Drug-Induced Liver Injuries. J. Prac Hepatol. 23 (02), 227-230. doi:10.3969/j.issn.1672-5069.2020.02.020

Wang, X., Zhao, S., Wang, Q., and Ma, A. (2018). Protective Effect of Bicyclol on Anti-tuberculosis Drug-Induced Hepatotoxicity: a Meta-Analysis. Chin. J. Dis. Control. Prev. 22 (04), 390-395. doi:10.16462/ j.cnki.zhjbkz.2018.04.016

Wang, Y., Wang, Z., Gao, M., Zhong, H., Chen, C., Yao, Y., et al. (2019). Efficacy and Safety of Magnesium Isoglycyrrhizinate Injection in Patients with Acute Drug-Induced Liver Injury: A Phase II Trial. Liver Int. 39 (11), 2102-2111. doi:10.1111/liv.14204

Wang, Y., Zhuge, Y. Z., Zhang, F., Zhang, M., Zhang, w., He, Q. B., et al. (2016). Treatment for Gynura Segetum Caused Hepatic Vein Occlusive Disease: a Single-center Retrospective Study. Chin. J. Dig. 36 (12), 811-815. doi:10.3760/cma.j.issn.0254-1432.2016.12.003

Warner, D. J., Chen, H., Cantin, L. D., Kenna, J. G., Stahl, S., Walker, C. L., et al. (2012). Mitigating the Inhibition of Human Bile Salt export Pump by Drugs: Opportunities provided by Physicochemical Property Modulation, In Silico Modeling, and Structural Modification. Drug Metab. Dispos 40 (12), 2332-2341. doi:10.1124/dmd.112.047068

Watkins, P. B. (2005). Idiosyncratic Liver Injury: Challenges and Approaches. Toxicol. Pathol. 33 (1), 1-5. doi:10.1080/01926230590888306

Wei, G., Bergquist, A., Broomé, U., Lindgren, S., Wallerstedt, S., Almer, S., et al. (2007). Acute Liver Failure in Sweden: Etiology and Outcome. J. Intern. Med. 262 (3), 393-401. doi:10.1111/j.1365-2796.2007.01818.x

Wree, A., Dechêne, A., Herzer, K., Hilgard, P., Syn, W. K., Gerken, G., et al. (2011). Steroid and Ursodesoxycholic Acid Combination Therapy in Severe Drug-Induced Liver Injury. Digestion 84 (1), 54-59. doi:10.1159/ 000322298

Wu, J., Xie, L. X., and Xiu, J. (20202020). Short-term Efficacy of Polyene Phosphatidylcholine and Bicyclol Combination in Treatment of Gastric Cancer Patients with Chemotherapy-Induced Liver Injury. J. Prac Hepatol. 23 (5), 666-669. doi:10.3969/j.issn.1672-5069.2020.05.016 
Xu, S. S., Qiu, L. X., Song, J. J., and Zhang, J. (20212021). Research Advances in Drug-Induced Vanishing Bile Duct Syndrome. J. Clin. Hepatol. 37 (8), 1979-1982. doi:10.3969/j.issn.1001-5256.2021.08.051

Ye, Y. J., Yu, J. P., Xu, Y. M., Wang, H., and Xie, Q. (2017). The Treatment Effect of Ursodeoxycholic Acid Combined with S-Adenosyl-L-Methionine on Drug-Induced Cholestatic Liver Injury. Chin. Hepatol. 22 (12), 1090-1093. doi:10.14000/ j.cnki.issn.1008-1704.2017.12.007

Yu, C. Y., Chang, L. C., Chen, L. W., Lee, T. S., Chien, R. N., Hsieh, M. F., et al. (2014). Peliosis Hepatis Complicated by portal Hypertension Following Renal Transplantation. World J. Gastroenterol. 20 (9), 2420-2425. doi:10.3748/wjg.v20.i9.2420

Yue, Y. C., Mao, Y. M., and Chen, C. W. (2017). Diagnosis and Treatment Guideline on Drug-Induced Liver Injury. J. Prac Hepatol. 1 (02), 257-274. doi:10.3969/j.issn.1672-5069.2017.02.039

Zhao, H., Lv, F. F., Sang, R. X., Wang, X. Z., Li, Z. H., and Xie, w. (2021). Clinical Effect of Silybin Capsule on Drug-Induced Liver Injury. Gansu Med. J. 40 (06), 490-493. doi:10.15975/j.cnki.gsyy.2021.06.003

Zhou, X., Yang, X., Sun, Y., and Li, X. (2015). Bicyclol versus Diammonium Glycyrrhizinate for the Treatment of Drug-Induced Hepatic Injury in Patients with Severe Psoriasis: a Randomized Controlled Trial. Chin. J. Dermatol. 48 (04), 245-247. doi:10.3760/cma.j.issn.0412-4030.2015.04.007

Conflict of Interest: The authors declare that the research was conducted in the absence of any commercial or financial relationships that could be construed as a potential conflict of interest.

Publisher's Note: All claims expressed in this article are solely those of the authors and do not necessarily represent those of their affiliated organizations, or those of the publisher, the editors and the reviewers. Any product that may be evaluated in this article, or claim that may be made by its manufacturer, is not guaranteed or endorsed by the publisher.

Copyright $\odot 2022 \mathrm{Li}, \mathrm{Luo}, \mathrm{Tao}$, Sun and Liu. This is an open-access article distributed under the terms of the Creative Commons Attribution License (CC BY). The use, distribution or reproduction in other forums is permitted, provided the original author $(s)$ and the copyright owner(s) are credited and that the original publication in this journal is cited, in accordance with accepted academic practice. No use, distribution or reproduction is permitted which does not comply with these terms. 